\title{
Application of the DCP model to a metastable racemate of mandelic acid
}

\author{
M. Pauchet and G. Coquerel \\ Unité de Croissance Cristalline et de Modélisation Moléculaire (UC $\left.{ }^{2}{ }^{2}\right)$ Sciences \\ et Méthodes Séparatives (SMS) UPRES EA 3233, IRCOF, Université de Rouen, \\ 76821 Mont-Saint-Aignan Cedex, France
}

\begin{abstract}
Mandelic acid is a chiral molecule often used as a resolving agent in the separation of enantiomers. To date, three different crystalline structures have been characterized: the pure enantiomer and two polymorphs of the racemic compound. However, the metastable racemic and the pure enantiomeric crystal structures share analogies. Because of these similarities, the DCP model has been applied to rebuild the metastable racemic compound by recombining a periodic fragment extracted from the crystal structure of the enantiomer. As computations reveal another very similar crystal lattice of the racemic compound, possible disorder and/or twining and/or polytypism are surmised.
\end{abstract}

\section{THE DCP MODEL}

The DCP (Derived Crystal Packing) ${ }^{1}$ model is a simple two-stepped procedure which allows to build new crystal structures starting from a known one. In a first step, a periodic fragment is extracted from the mother phase. It can be either a one-dimensional or a bidimensional part of the structure (respectively one parameter and one direction or two parameters and two directions are kept from the unit cell) and can include some of its symmetry operators.

A new three-dimensional crystal structure is built from this periodic fragment by adding symmetry operators consistent with the International Tables of Crystallography. This is performed with SYBYL Software $^{2}$, by using a dedicated homemade program that computes the energies of interactions in the structure with respect to the nature and the location of the symmetry operators. In this study, molecules are used as rigid bodies. Therefore, in a first approximation, only steric interaction energies are taken into account. They are based on a Lennard-Jones attractive and repulsive contribution from the Tripos Forcefield ${ }^{2}$. The computed energies are related to the close-packing principle: the highest density will determine the most probable structure between several energetically similar daughter phases. Depending on the nature of the added symmetry operators and their locations with reference to the periodic fragment, several daughter phases of different spacegroups can thus be obtained by starting from the same mother phase. 


\section{STRUCTURES OF MANDELIC ACID}

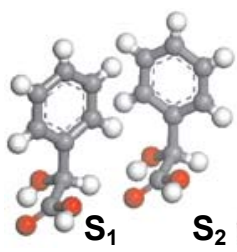

Figure 1. Asymmetric unit of $\mathrm{S}$ mandelic acid.
$\mathrm{S}$ mandelic acid crystallizes in the spacegroup $\mathrm{P} 2{ }_{1}$. Its crystallographic parameters are: $\mathrm{a}_{\text {pures }}=8.629$ $\AA, \mathrm{b}_{\text {pures }}=5.861 \AA, \mathrm{c}_{\text {pures }}=15.185 \AA$ and $\mathrm{E}_{\text {pures }}=$ $102.76^{\circ} 3,4$, Its asymmetric unit includes two molecules $\left(S_{1}\right.$ and $S_{2}$ in Figure 1$)$, with very similar conformations. Comparisons between the different torsion angles show that molecules mainly differ by the orientation of the phenyl groups, due to pi-stacking (Table 1).

The structure of metastable mandelic acid (hereafter racfII) has been solved by Fisher et al. ${ }^{6}$. They highlighted similarities with the structure of the mandelic acid $\mathrm{S}$ enantiomer. Indeed, both structures share crystallographic parameters: racfII belongs to the spacegroup $\mathrm{P} 2{ }_{1} / \mathrm{c}$ with $\mathrm{a}_{\text {racfII }}=5.847 \AA$, $\mathrm{b}_{\text {racfII }}=$ $29.241 \AA, c_{\text {racfII }}=8.723 \AA$ and $\mathrm{E}_{\text {racfII }}=92.165^{\circ}$. Its asymmetric unit also includes two molecules which differ by the orientation of their phenyl groups (Table 1). Moreover, these orientations can be related to those of the pure enantiomeric form. The comparisons focus attention on the close conformations between on the one hand $S_{1 \text { pures }}$ and $S_{1 \text { racfII }}$, and on the other hand $S_{2 \text { pures }}$ and $S_{2 \text { racfII. }}$.

Table 1. Torsion angles of pure $S$ and racemic metastable mandelic acid.

\begin{tabular}{|l|l|l|l|l|}
\cline { 2 - 5 } \multicolumn{1}{c|}{} & \multicolumn{2}{c|}{ Pure S } & \multicolumn{2}{c|}{ Metastable racemate } \\
\cline { 2 - 5 } \multicolumn{1}{c|}{} & Molecule $\mathrm{S}_{1}$ & Molecule $\mathrm{S}_{2}$ & Molecule $\mathrm{S}_{1}$ & Molecule $\mathrm{S}_{2}$ \\
\hline C1-C2-C3-C4 & $88.581^{\circ}$ & $139.177^{\circ}$ & $94.297^{\circ}$ & $140.785^{\circ}$ \\
\hline O3-C2-C3-C4 & $-149.56^{\circ}$ & $-98.232^{\circ}$ & $-143.192^{\circ}$ & $-97.094^{\circ}$ \\
\hline O1-C1-C2-O3 & $176.777^{\circ}$ & $179.815^{\circ}$ & $177.247^{\circ}$ & $179.891^{\circ}$ \\
\hline
\end{tabular}

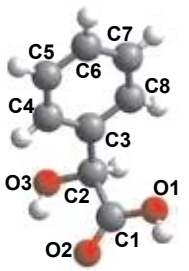

The lattice of the pure $\mathrm{S}$ form includes H-bond networks due to interactions between both acid moiety and hydroxyde groups. Two H-bond networks can be consequently distinguished, each one containing either $S_{1}$ or $S_{2}$ and running along $b_{\text {pures }}$ (and including the $2_{1}$ screw axis). Although racfII also forms $\mathrm{H}$-bond networks (running along $\mathrm{a}_{\text {racfII }} \sim \mathrm{b}_{\text {pures }}$ ) analogous to those formed by the structure of the pure enantiomeric form, it differs by the participation of both enantiomers. Two parallel H-bond networks can be distinguished by the nature of the molecules involved: one with $S_{1}$ and $R_{1}$ and the other with $\mathrm{S}_{2}$ and $\mathrm{R}_{2}$.

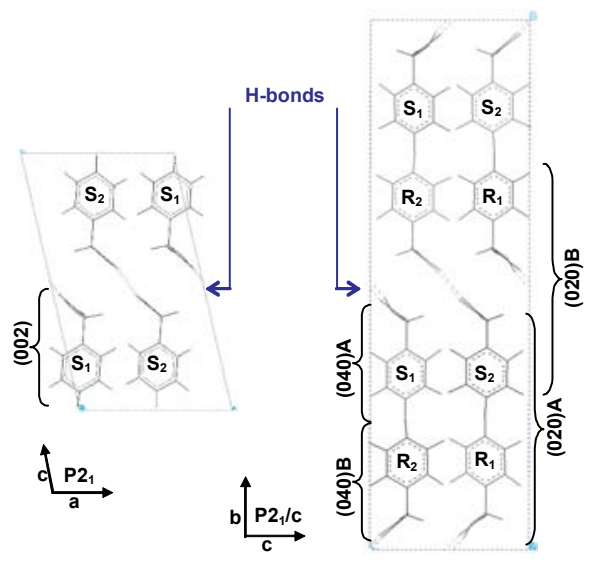

Figure 2. Unit cells of (S) and (RS)-metastable mandelic acid. 
Figure 2 shows that both structures are composed of pure enantiomeric layers including the two molecules of the asymmetric unit ((002) for the pure enantiomeric form and (040)A and (040)B for racfII), stacked along the third direction.

\section{APPLICATION OF THE DCP MODEL}

\subsection{Choice of the Periodic Fragment}

The nature of the Periodic Fragment (PF hereafter) extracted from the mother phase (pure $\mathrm{S}$ form) is the cornerstone of the procedure. In the present case, the daughter phase is expected to have two molecules in its asymmetric unit. Therefore the PF must include two independent molecules, i.e. $\mathrm{S}_{1 \text { pures }}$ and $\mathrm{S}_{2 \text { pures. }}$.

The comparisons between crystallographic parameters of pure $\mathrm{S}$ and racfII seem to indicate that $(002)_{\text {pures }}$ and (040) $\mathrm{A}_{\text {racfII }}$ are almost identical.

The study of the crystal graph ${ }^{7}$ of the structure of pure $S$ reveals strong interations in $(002)_{\text {pures }}$ slices which seems to designate this slice as a good candidate for the reconstruction (a bi-dimensional (001) $\mathrm{PF}$ would lead to a structure with two $\mathrm{S}$ homochiral layers alternating with two $\mathrm{R}$ homochiral layers). However the $(002)_{\text {pures }}$ slice has a fixed angle of $90^{\circ}$, whereas the angle is expected to be slightly different in the daughter phase $\left(E_{\text {racfII }}=92.165^{\circ}\right)$. Finally, the selected PF is a [100] ribbon of $-S_{1}-S_{2}-S_{1}-S_{2^{-}}$, along $a_{\text {pures }}$ direction. This parameter gives the new $c_{\text {racfII }}$ parameter.

\subsection{Addition of the symmetry operators}

\subsubsection{Overview of the regeneration of the daughter phase}

The generation of the daughter phase is performed in three consecutive steps. First, a slice of the final structure is produced from the initial PF by adding a translation. A glide plane parallel to the slice is then added generating a new slice of double thickness, and finally a $2_{1}$ screw axis orthogonal to this slice yields the three-dimensional daughter phase.

\subsubsection{Generation of a two-dimensional periodic fragment}

The $[100]_{\text {pures }}$ ribbon is docked onto another one. Energies are recorded according to the shift along the $c_{\text {racfII }}$ direction and the relative distance between both PF's. $a_{\text {racfII }}$ and $E_{\text {racfII }}$ are thus investigated at a time. The results of the docking are presented on Figure. Respecting the close-packing principle means that the shortest distance between ribbons has to be found. $E_{\text {racfII }}$ and $a_{\text {racfII }}$ respectively ranging from $92.4^{\circ}$ to $96.3^{\circ}$ and from $5.87 \AA$ to $5.90 \AA$ fulfill this condition. The best result is obtained for $\mathrm{E}=92.4^{\circ}$ and $\mathrm{a}=5.87 \AA$, yielding the $(040) \mathrm{A}_{\text {racfII }}$ slice

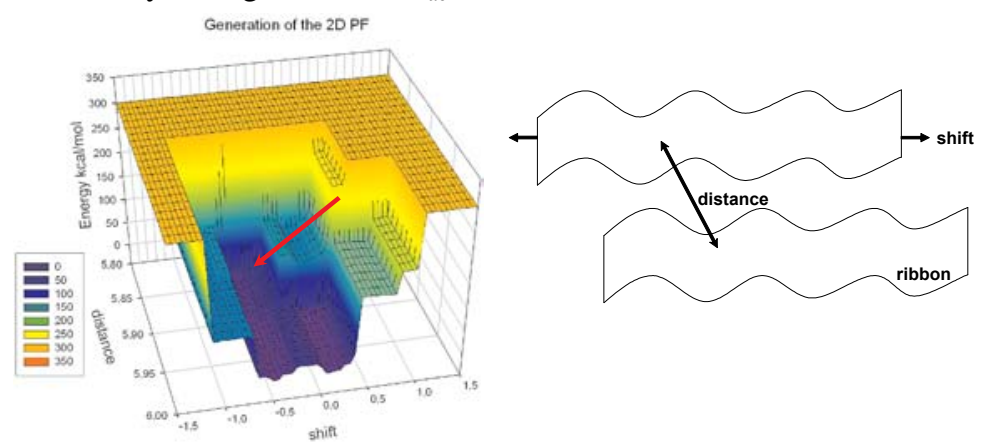

Figure 3. Generation of a slice from the initial PF. 


\subsubsection{Addition of the glide plane}

Heterochiral (020) $\mathrm{A}_{\text {racfII }}$ slices are built by adding a glide mirror (c) parallel to $\mathrm{a}_{\text {racfII }}$ and $\mathrm{c}_{\text {racfII }}$. Since the associated translation is defined by the nature of this operator (i.e. half of the $c_{\text {racfII }}$ parameter), only one degree of freedom remains: the distance between the glide plane and the slice. This distance sets the thickness of the heterochiral $(020) \mathrm{A}_{\text {racfII }}$ slice (see Figure 4).
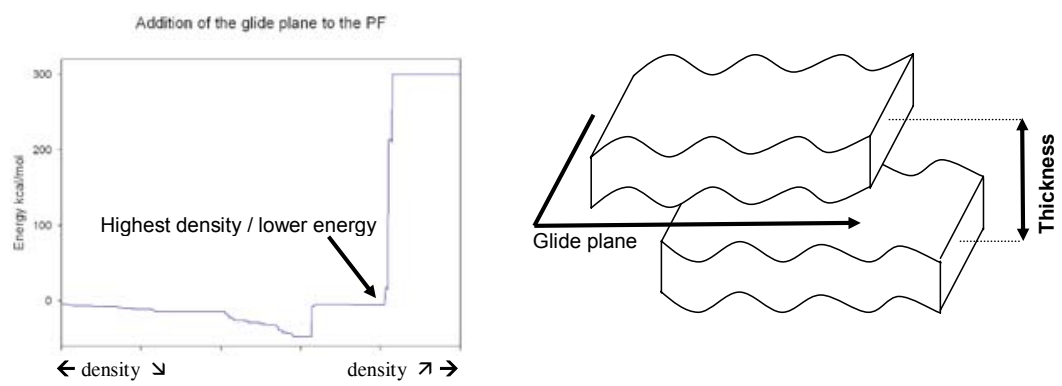

Figure 4. Addition of a glide plane (c) to the slice.

\subsubsection{Addition of the $2_{1}$ screw axis}

The final stage of the reconstruction consists in adding a new symmetry operator to access to a threedimensional periodic array. A $2_{1}$ screw axis is consequently added orthogonally to the (020) $\mathrm{A}_{\text {racfII }}$ slice. The docking involves three degrees of freedom. The length of the associated translation defines the third crystallographic parameter (length of translation $=1 / 2 b_{\text {racfII }}$ ). Since the previously added glide plane does not set any restriction concerning the location of this screw axis, both $a_{\text {racfII }}$ and $c_{\text {racfII }}$ directions have to be investigated. 3-dimensional graphs showing the interaction versus the location of the screw axis are plotted for a set of $b_{\text {racfII }}$ values (Figure 5).
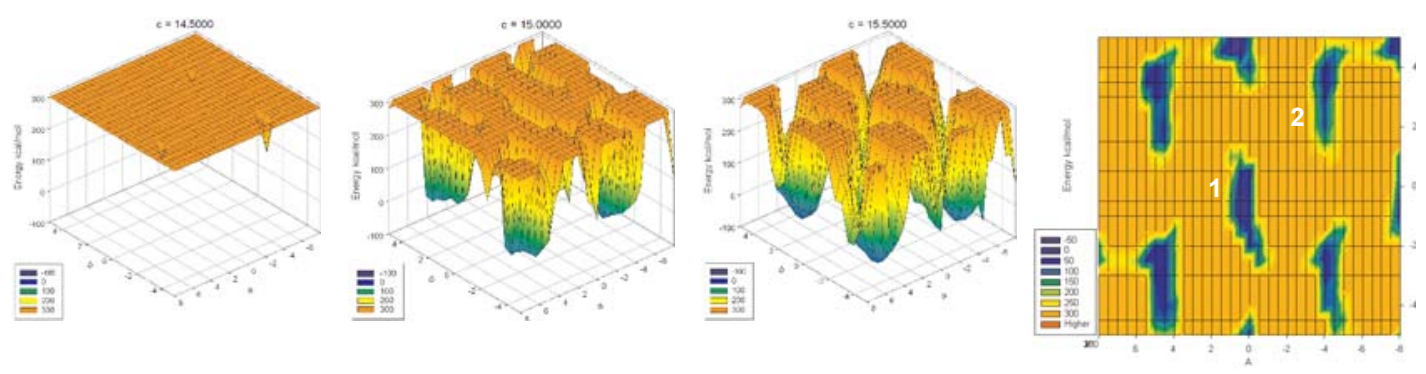

Figure 5. Interaction energies during the docking.

Unexpectedly, the results of this docking show two (periodically repeated) locations for the $2_{1}$ screw axis leading, thus, to two different daughter phases. They appear on the graphs as two distinct hollows (right of the Figure 5). Two daughter phases (DP1 and DP2) with the same crystallographic parameters can therefore be built from these results: $a_{\text {racfII }}=5.87 \AA, b_{\text {racfII }}=29.60 \AA$, $\mathrm{c}_{\text {racfII }}=8.629 \AA$ and $\beta_{\text {racfII }}=92.43^{\circ}$.

\subsection{Results and discussion}

The distinction between the locations of the screw axes in DP1 and DP2 results from the difference in the position of the molecules inside the unit-cell (Figure 6). However, half of the structures are superimposable since they were built from the same $(020) \mathrm{A}_{\text {racfII }}$ slice. The daughter phases are compared with the experimental racfII structure. DP1 matches with racfII with a good agreement. 
DP2 has only one halve of the unit-cell in common with racfII; in the other halve the molecules 1 and 2 are inverted (see schematic representation in Figure 6 ).
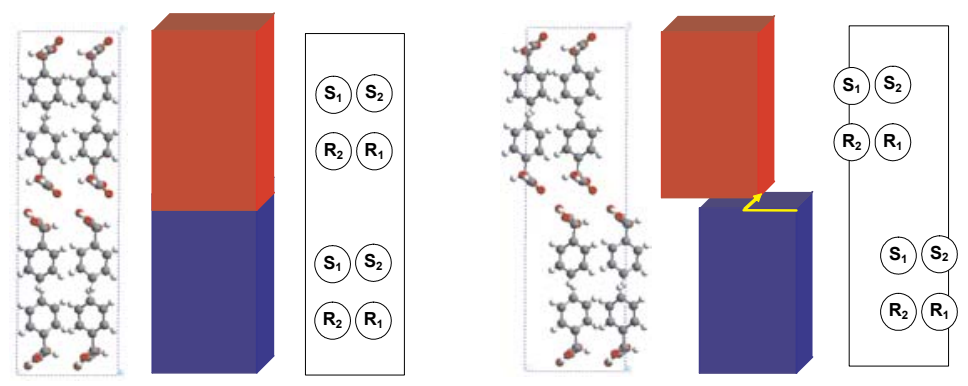

Figure 6. Daughter phases DP1 (left) and DP2 (right).

In order to assess the probability of existence of the second daughter phase, both structures were first minimized by using Cerius ${ }^{2,8}$. The peculiar orientation of the phenyl groups is preserved by assigning a restraint on $\mathrm{C} 1-\mathrm{C} 2-\mathrm{C} 3-\mathrm{C} 4$ torsion angle. The computed energies after minimization only diverge by a few $\mathrm{kcal} / \mathrm{mol}$. This similarity may be explained by the H-bond networks that link the (020) slices.

Whereas the orientation of the phenyl groups differ between molecules 1 and 2, the functionalized chain ( $\mathrm{HO}-\mathrm{CH}-\mathrm{COOH})$ keeps the same spatial arrangement. Switching between the molecules shouldn't significantly change the positions of the atoms involved in the formation of the H-bond networks.

XRPD patterns have been simulated in order to discriminate both phases. Diffraction peaks are at the same $2 \mathrm{~T}$ values, with variations in intensity. However, since the crystallographic parameters and the space groups of DP1 and DP2 are identical, the variations on intensity of the diffraction peaks are very sensitive to the

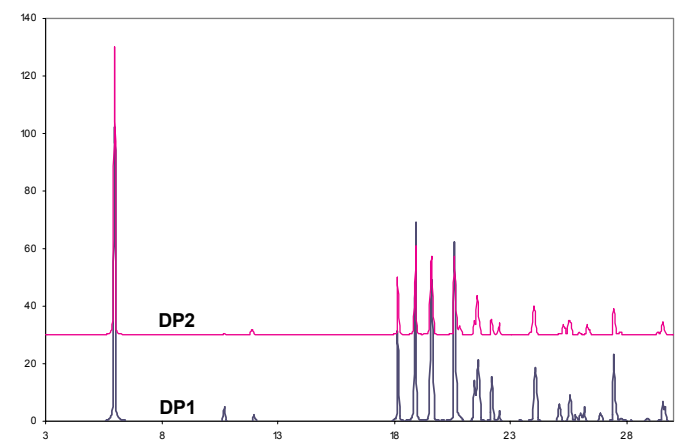

Figure 7. Simulated XRPD patterns of DP1 and DP2.

\section{CONCLUSION}

Starting from the structure of the pure enantiomer only, the DCP model allows to build the crystal structure of the metastable racemic mandelic acid. However, at least another structure with similar energy and packing is predicted by the model. Further work will be devoted to spot possible twins, disorder, polymorphic and/or polytypic structures.

\section{Acknowledgements}

Interreg IIIA network is thanked for its continuous support. 


\section{References}

[1] Gervais C., Coquerel G., Acta Cryst. B58 (2002) 662-672

[2] SYBYL 6.9.0, Tripos Associates: St Louis, MO, USA, web site : www.tripos.com

[3] Cameron T.S., Duffin M. Cryst. Struct. Commun., 3 (1974) 539-541

[4] Wei K.-T., Ward D.L. Acta Cryst B33 (1977) 797-800

[5] Patil A.O., Pennington W.T., Paul I.C., Curtin D.Y., Dykstra C.E. J. Am. Chem. Soc. 109 (1987) 1529-1535

[6] Fisher A., Profir V. M. Acta Cryst. E59 (2003) 1113-1116

[7] Grimbergen R. F. P., Meekes H., Bennema P., Strom C. S., Vogels L. J. P. Acta Cryst A54 (1998) 491-500

[8] Cerius $^{2}$, Accelrys Inc., version 4.8.1, Cambridge, U.K., web site : www.accelrys.com 